\title{
EXTENDED MID-INFRARED AROMATIC FEATURE EMISSION IN M82
}

\author{
C. W. Engelbracht, ${ }^{1}$ P. Kundurthy, ${ }^{1}$ K. D. Gordon, ${ }^{1}$ G. H. Rieke, ${ }^{1}$ R. C. Kennicutt, ${ }^{1,2}$ J.-D. T. Smith, ${ }^{1}$ M. W. Regan, ${ }^{3}$ \\ D. Makovoz, ${ }^{4}$ M. Sosey, ${ }^{3}$ B. T. Draine, ${ }^{5}$ G. Helou, ${ }^{4}$ L. Armus, ${ }^{4}$ D. Calzetti, ${ }^{3}$ M. Meyer, ${ }^{3}$ G. J. Bendo, ${ }^{6}$ F. Walter, ${ }^{7}$ \\ D. Hollenbach, ${ }^{8}$ J. M. Cannon, ${ }^{7}$ E. J. Murphy, ${ }^{9}$ D. A. Dale, ${ }^{10}$ B. A. Buckalew, ${ }^{4}$ and K. Sheth ${ }^{4}$ \\ Received 2006 February 2; accepted 2006 March 27; published 2006 April 17
}

\begin{abstract}
We present new images (ground-based optical and mid-infrared [MIR] from the Spitzer Space Telescope) and spectra (from Spitzer) of the archetypal starburst galaxy M82. The Spitzer data show that the MIR emission extends at least $6 \mathrm{kpc}$ along the minor axis of the galaxy. We use the optical and infrared data to demonstrate that the extended emission is dominated by emission from dust. The colors of the MIR emission and the spectra indicate that there is a strong component of aromatic feature emission (the MIR features commonly attributed to polycyclic aromatic hydrocarbons). The dust continuum and aromatic feature emission are both strong in the well-known superwind region of this galaxy; clearly, the carrier of the aromatic features can survive in close proximity to the wind, far from the plane of the galaxy. We also see significant emission by dust well outside the superwind region, providing the clearest picture to date of the dust distribution in the halo of this galaxy.
\end{abstract}

Subject headings: dust, extinction — galaxies: individual (M82) — galaxies: ISM — galaxies: peculiar — galaxies: starburst

\section{INTRODUCTION}

M82 is a nearby starburst galaxy in the M81 Group and perhaps the most well studied member of the starburst class. It has a high luminosity $\left(5 \times 10^{10} L_{\odot}\right.$; McLeod et al. 1993), most of which is emitted in the infrared. It is the brightest farinfrared (FIR) extragalactic source in the sky after the Magellanic Clouds. It was one of the first galaxies to be identified as a starburst galaxy (Rieke et al. 1980). As an archetypal starburst galaxy, M82 has been observed often and over a wide range of wavelengths. An incomplete sampling of recent studies that can be used as a guide to previous observations includes X-ray (Strickland et al. 2004), ultraviolet (Hoopes et al. 2005), optical and near-infrared (NIR) (McCrady et al. 2005; AlonsoHerrero et al. 2003), mid-infrared (MIR) (Förster Schreiber et al. 2003b), FIR (Colbert et al. 1999), and radio (RodríguezRico et al. 2004). The galaxy is also a popular target for stellar population modeling efforts (e.g., Rieke et al. 1980, 1993; Satyapal et al. 1997; Förster Schreiber et al. 2003a). M82 is interacting with the spiral galaxy M81 and the nearby dwarf starburst galaxy NGC 3077, as evidenced by the H I tails linking the three galaxies (Yun et al. 1994). M81 lies at a distance of 3.6 Mpc (Freedman et al. 2001), and we adopt this distance for M82 as well.

\footnotetext{
${ }^{1}$ Steward Observatory, University of Arizona, 933 North Cherry Avenue, Tucson, AZ 85721; cengelbracht@as.arizona.edu.

${ }^{2}$ Institute of Astronomy, University of Cambridge, Madingley Road, Cambridge CB3 0HA, UK.

${ }^{3}$ Space Telescope Science Institute, 3700 San Martin Drive, Baltimore, MD 21218.

${ }^{4}$ Spitzer Science Center, Mail Code 220-6, California Institute of Technology, 1200 East California Boulevard, Pasadena, CA 91125.

${ }^{5}$ Princeton University Observatory, Peyton Hall, Ivy Lane, Princeton, NJ 08544.

${ }^{6}$ Astrophysics Group, Blackett Laboratory, Imperial College of Science, Technology and Medicine, Prince Consort Road, London SW7 2BW, UK.

${ }^{7}$ Max-Planck-Institut für Astronomie, Königstuhl 17, D-69117 Heidelberg, Germany.

${ }^{8}$ NASA Ames Research Center, Moffett Field, CA 94035.

${ }^{9}$ Department of Astronomy, Yale University, P.O. Box 208101, New Haven, CT 06520.

${ }^{10}$ Department of Physics and Astronomy, University of Wyoming, Laramie, WY 82071-3905.
}

M82 is a so-called superwind galaxy, in which the central starburst drives a mass outflow perpendicular to the plane of the galaxy, as discussed in detail by Heckman et al. (1990), Shopbell \& Bland-Hawthorn (1998), and Martin (1998). The superwind emanates from the central starburst region and is most evident in X-ray (e.g., Strickland et al. 2004) and $\mathrm{H} \alpha$ (e.g., Lehnert et al. 1999) emission from the gaseous component of the wind. There is also dust in the superwind region, as observed in reflection (Hoopes et al. 2005 and references therein), absorption (Heckman et al. 2000), and emission from cool grains (Alton et al. 1999). This work not only provides the first direct detection of the warm dust component throughout the superwind region, but also demonstrates that the dust is distributed all around the galaxy, well beyond the cone defined by the superwind.

Galaxies not dominated by an active nucleus in the MIR generally show a series of strong, broad emission features arising from aromatic hydrocarbons (see Laurent et al. 2000; Lu et al. 2003; J.-D. T. Smith et al. 2006, in preparation) if the galaxy metallicity is above $\sim \frac{1}{5}$ solar (Engelbracht et al. 2005). These features are often attributed to polycyclic aromatic hydrocarbons (PAHs), but since the identification of the carrier remains controversial, we will use the more generic term aromatic feature emission (AFE). The features are strong in the high surface brightness central regions of M82, where they have been observed both from the ground (Roche et al. 1991; Normand et al. 1995) and from space (Sturm et al. 2000; Förster Schreiber et al. 2003b). Here we demonstrate that these features can also be found throughout the halo, up to $6 \mathrm{kpc}$ from the plane of the galaxy.

\section{OBSERVATIONS AND DATA REDUCTION}

The data presented in this Letter were obtained as part of the Spitzer Infrared Nearby Galaxy Survey (SINGS; Kennicutt et al. 2003). We employ here a subset of the suite of SINGS data that allows us to trace stars ( $I$ band), ionized gas (continuum-subtracted $\mathrm{H} \alpha$ ), and hot dust (3-9 $\mu \mathrm{m}$ images from IRAC, a $24 \mu \mathrm{m}$ image from MIPS, and 14-36 $\mu \mathrm{m}$ spectra from IRS) in M82. To minimize detector artifacts due to the high surface 
brightness of the central starburst, the IRAC data were obtained in separate maps for the inner and outer regions. Both the IRAC and MIPS data required custom reduction procedures to produce maps free of detector artifacts. The images are shown in Figure 1 (Plate 1). Note that the $24 \mu \mathrm{m}$ image has strong diffraction spikes due to the extremely bright nucleus.

We also obtained a spectral map with IRS in a $1^{\prime}$-wide strip along the minor axis of the galaxy, centered on the eastern portion of the disk. Spectra were extracted from four regions (indicated in Fig. 1c) where a high signal-to-noise ratio could be obtained. These data are shown in Figure 2, where we highlight the portion of the spectrum around the aromatic features; the full spectrum of one region is shown for reference. The resolution $(\lambda / \Delta \lambda)$ of these data is $\sim 60-130$.

We performed photometry on the images at positions indicated in Figure 1e. The regions were chosen to sample a variety of environments, from high surface brightness disk regions to diffuse regions well outside the disk of the galaxy. Colors indicate different types of regions ( $\mathrm{red}$, disk; blue, edge of disk; light blue, overlap with IRS spectra; green, diffuse filament). Most of the apertures are circular, with a radius of 4 ".25 or $18^{\prime \prime}$ (which can be compared with the half-width at half-maximum of the IRAC and MIPS beams of $\sim 1^{\prime \prime}$ and $3^{\prime \prime}$, respectively) chosen to encompass typical emission regions without overlapping. The regions measured contain mostly diffuse emission, and so the only aperture corrections made were to the IRAC surface brightness (Reach et al. 2005). To minimize the impact of the bright nucleus on our $24 \mu \mathrm{m}$ measurements, we first subtracted a simple model of the nuclear region (based on a $20 \mu \mathrm{m}$ map by Rieke et al. [1980]), convolved with the $24 \mu \mathrm{m}$ point-spread function generated using observations of HD 44179 (Spitzer AOR key 9548800) and scaled to match the observed emission in the nonsaturated regions of the galaxy. The subtracted image is shown in Figure 3 (Plate 2). In all cases, a background value measured in regions free of galaxy emission, outside the area shown in Figure 1, was subtracted from the measurements.

\section{RESULTS}

\subsection{Morphology}

The morphology of M82 is a strong function of wavelength. The optical broadband images are dominated by an edge-on disk with a prominent dust absorption lane near the center. This dust lane conceals the nucleus, near the peak of the starburst activity. This becomes obvious at longer wavelengths less affected by extinction: in the IRAC band 1 image at $3.6 \mu \mathrm{m}$, the prominent disk is more symmetric and dominated by a central bright source, similar to ground-based $K$-band images (e.g., McLeod et al. 1993; Jarrett et al. 2003). It is in IRAC band 1, though, that we begin to see a departure from the picture obtained from the optical images - there is filamentary emission perpendicular to the disk of the galaxy that extends for several arcminutes (an arcminute is roughly a kiloparsec at the distance of M82). This emission is more prominent at $4.5 \mu \mathrm{m}$ and becomes a dominant characteristic of the image by $8.0 \mu \mathrm{m}$, where filaments are detected a full $6 \mathrm{kpc}$ from the disk of the galaxy. The extended emission observed in the IRAC bands accounts for a significant fraction of the total: about one-third of the 4.5 and $8.0 \mu \mathrm{m}$ emission arises outside the central $1 \times 0.6 \mathrm{kpc}$, while the equivalent fraction at $24 \mu \mathrm{m}$ is about one-fifth (where we have used the model described in $\S 2$ to account for the saturated region). The diffuse extraplanar MIR emission evokes the $\mathrm{H} \alpha$ image, which is dominated by a biconical structure that
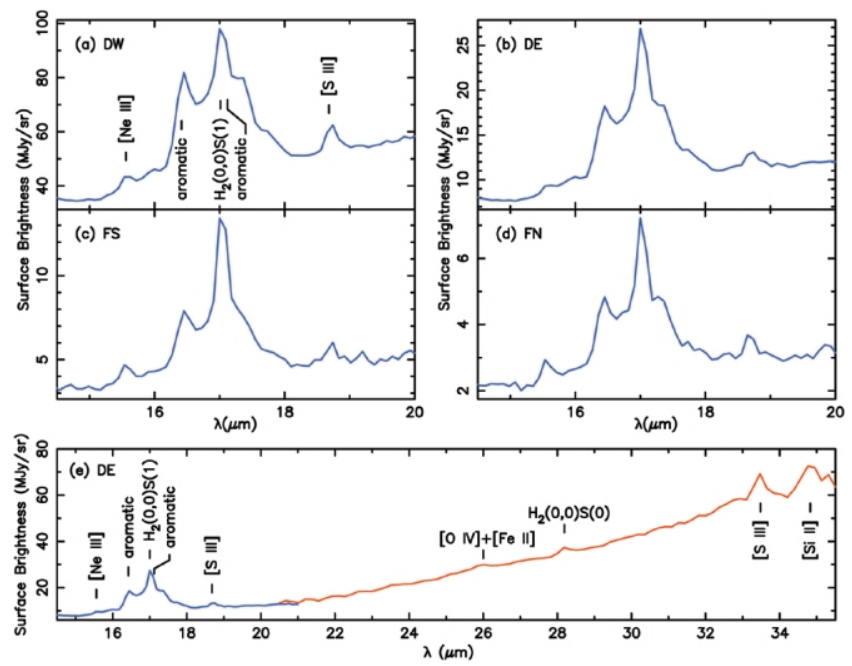

FIG. 2.-Rest-wavelength IRS spectra of selected regions of M82. (a-d) The portion of the spectrum around the aromatic features in the western disk region (DW), the eastern disk region (DE), the south filament (FS), and the north filament $(\mathrm{FN})$, respectively. (e) The full spectrum of the eastern disk region, where blue indicates the spectrum extracted from long-low order 2 while red indicates the spectrum extracted from long-low order 1.

defines the superwind. In the superwind region, the morphology of the MIR emission is similar to that of the $\mathrm{H} \alpha$ emission and other tracers of the superwind such as UV (Hoopes et al. 2005) and X-rays (Strickland et al. 2004), but the MIR images differ in that the emission is bright all around the galaxy rather than being dominated by a cone perpendicular to the plane. Although the morphology at $24 \mu \mathrm{m}$ is dominated by the very bright central source and the accompanying diffraction spikes, between the diffraction spikes the diffuse emission is very similar to the $8 \mu \mathrm{m}$ image, as shown in Figure 3. Clearly, the infrared images reveal a different component of the galaxy than the optical or superwind tracers.

The extent of the MIR emission is highly unusual. Inspection of other images in the SINGS sample or in published Spitzer samples (e.g., Pahre et al. 2004) does not reveal any other galaxy with similar characteristics. We find MIR emission extending along the minor axis of the galaxy from all parts of the disk, not just the superwind region. This suggests that there is a material in the halo of this galaxy that is capable of significant emission in the MIR. The material may have been ejected from the disk over time by means of a galactic fountain process similar to, but more vigorous than, that in NGC 5907 (Irwin \& Madden 2006), but the unique nature of the MIR emission from the halo of M82 argues against such a general process. More likely, the interaction with M81 may have ejected material over a shorter time by triggering a starburst (e.g., de Grijs et al. 2001; McLeod et al. 1993). Some of this material may be entrained in the superwind as in NGC 253 (TacconiGarman et al. 2005), but it is likely much of the MIR emission traces a medium with which the superwind is interacting as it expands into the halo.

\subsection{Components of Extraplanar MIR Emission}

We turn now to elucidating the nature of the extraplanar MIR emission: dust emission, starlight, or line emission, and their relative contributions. We compute the stellar contribution at MIR wavelengths by scaling a short-wavelength measurement (e.g., $I$-band or $3.6 \mu \mathrm{m}$ ) by the appropriate factor, derived 
using stellar population synthesis models. In the disk, where the extinction is high and the equivalent width of the $3.3 \mu \mathrm{m}$ aromatic feature is low (cf. Förster Schreiber et al. 2001), we scale the $3.6 \mu \mathrm{m}$ band by factors of 0.57 and 0.23 at 4.5 and $8.0 \mu \mathrm{m}$, respectively (cf. Helou et al. 2004; Engelbracht et al. 2005), to compute the stellar contribution. In the halo, where the extinction is low and the equivalent width of the $3.3 \mu \mathrm{m}$ aromatic feature is possibly high (as evidenced by the extraplanar filaments in the $3.6 \mu \mathrm{m}$ image shown in Fig. 1), we scale the $I$ band by factors of 0.18 and 0.075 (as computed using the model by Leitherer et al. [1999], about a factor of 2 lower than we obtain using the model by Bruzual \& Charlot [2003]) at 4.5 and $8.0 \mu \mathrm{m}$, respectively. We find that the stellar contribution to the emission well outside the plane of the galaxy is no more than a few percent in any IRAC band. In all cases, only a negligible fraction of the $24 \mu \mathrm{m}$ emission is stellar.

The only prominent spectral lines typically found in galaxies that fall in the MIR bands we consider here that are not due to AFE are both found in the IRAC $4.5 \mu \mathrm{m}$ band: $\mathrm{Br} \alpha$ at $4.05 \mu \mathrm{m}$ (observed in the nucleus of M82; Förster Schreiber et al. 2001) and multiple $\mathrm{H}_{2}$ lines (e.g., Noriega-Crespo et al. 2004; B. T. Draine 2006, in preparation). We compute the $\operatorname{Br} \alpha$ contribution by scaling the observed $\mathrm{H} \alpha$ emission by the hydrogen recombination line intensities computed by Storey \& Hummer (1995). This estimate is subject to uncertain extinction corrections in the dusty disk of the galaxy, but it should be robust for the extraplanar emission. We find that $\mathrm{Br} \alpha$ contributes no more than a few percent of the total $4.5 \mu \mathrm{m}$ emission in any of our photometric regions. We estimate the strength of the $\mathrm{H}_{2}$ lines in the $4.5 \mu \mathrm{m}$ band by scaling the strength of the $(0,0) S(0)$ line according to the photodissociation region models by Draine \& Bertoldi (1996). We find that the possible $\mathrm{H}_{2}$ contribution at $4.5 \mu \mathrm{m}$ covers a wide range, from $\sim 0.001 \%$ to nearly $30 \%$.

Thus, the contribution to the extraplanar emission from starlight and hydrogen emission lines is insignificant (except perhaps the $\mathrm{H}_{2}$ contribution at $4.5 \mu \mathrm{m}$ ). We conclude that the extraplanar MIR emission is dominated by dust. At $3.6 \mu \mathrm{m}$, the emission is likely a combination of AFE and hot dust (see Helou et al. 2000; Lu et al. 2003). We turn to broadband colors and spectroscopy to determine what kind of dust (specifically, continuum or AFE) is contributing to the $8 \mu \mathrm{m}$ band, starting with the diagnostic diagram developed in Engelbracht et al. (2005). Our photometric regions are plotted in Figure 4, where one can see that all the points fall in the part of the plot where the $8 \mu \mathrm{m}$ band is dominated by AFE. This is consistent with our spectra, which show that the $17 \mu \mathrm{m}$ AFE feature discovered by Smith et al. (2004) and Werner et al. (2004) remains strong even in the extraplanar regions (see Fig. 2). The strength of the $17 \mu \mathrm{m}$ complex, scaled by the average $(8 \mu \mathrm{m}$ complex $) /$ (17 $\mu \mathrm{m}$ complex) ratio of SINGS galaxies (J.-D. T. Smith et al. 2006, in preparation), is sufficient to explain the IRAC band 4 emission in the regions where we extracted spectra. The fact that the dust in the outflow is readily detected in the IRAC bands and at $24 \mu \mathrm{m}$ supports the $\sim 37 \mathrm{~K}$ "warm" model of Alton et al. (1999) for the extraplanar dust. This model predicts a total outflowing dust mass of $\sim 10^{6} M_{\odot}$, which they show is roughly compatible with other estimates (e.g., from reddening of background galaxies).

\section{CONCLUSIONS}

We have presented new Spitzer images and spectra of the starburst galaxy M82 in the MIR and new ground-based optical

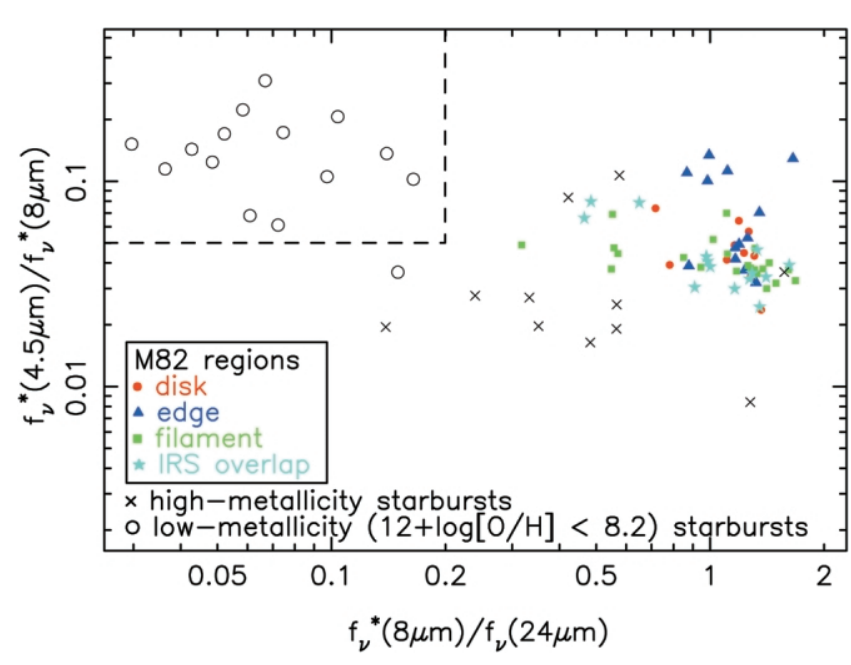

FIG. 4.-Colors of selected regions of M82. Asterisks on $f_{\nu}$ indicate that stellar emission has been subtracted as described in the text. Also plotted are the starburst galaxy points from Engelbracht et al. (2005). The dashed line encloses the color space found in that work to indicate a lack of AFE.

imaging, including a deep $\mathrm{H} \alpha$ map. The Spitzer data demonstrate that the galaxy has copious MIR emission well outside the plane of the galaxy. At $8.0 \mu \mathrm{m}$, this emission extends to $6 \mathrm{kpc}$ from the plane, and the emission outside the central $1 \times$ $0.6 \mathrm{kpc}$ accounts for about one-third of the total flux. We use the optical data to quantify the small contributions to the MIR bands from starlight and line emission and conclude that most of the MIR emission must be due to dust. At $24 \mu \mathrm{m}$, this emission is due to a featureless continuum arising from warm dust grains. At 3.6 and $8.0 \mu \mathrm{m}$, the colors and the spectra confirm a significant contribution by aromatic features, while the $4.5 \mu \mathrm{m}$ emission may be due to a combination of hot dust and $\mathrm{H}_{2}$ emission.

The MIR emission, especially at $8.0 \mu \mathrm{m}$, is filamentary and extends in all directions and from all parts of the disk of the galaxy. The fact that significant emission by dust is detected outside the well-known superwind emanating from this galaxy suggests that some process prior to the current starburst resulted in the expulsion of dust from all parts of the disk. The superwind is expanding into this dusty medium, and both small dust particles and the carrier of the AFE appear to survive the interaction. Some dust may be entrained in the wind, and dust is found well beyond the radius where gas is thought to be escaping the galaxy (cf. Shopbell \& Bland-Hawthorn 1998)if this dust is escaping as well, the carrier of the AFE is entering the intergalactic medium.

There may be alternative possibilities to the MIR-emitting dust being entrained in the superwind and sharing its high velocity. The superwind region is complex, containing, in addition to the hot plasma, shock-heated [Fe II] emission (AlonsoHerrero et al. 2003), molecular gas (Nakai et al. 1987; Loiseau et al. 1990; Walter et al. 2002) including cool $\mathrm{H}_{2}$ emitting from the low-lying transitions seen at 17 and $28 \mu \mathrm{m}$ (this work), dust grains seen here at $24 \mu \mathrm{m}$ and in the submillimeter (Alton et al. 1999), and aromatic molecules (this work). It is conceivable that some of the dust or aromatic molecules form in situ, or that radiation pressure aids the escape of small particles from the galaxy.

We thank the anonymous referee for a constructive report that improved this Letter. This work is based in part on ob- 
servations made with the Spitzer Space Telescope, which is operated by the Jet Propulsion Laboratory, California Institute of Technology, under NASA contract 1407. Support for this work, part of the Spitzer Space Telescope Legacy Science Program, was provided by NASA through contracts 1224769 and 1255094 issued by JPL/Caltech.

\section{REFERENCES}

Alonso-Herrero, A., Rieke, G. H., Rieke, M. J., \& Kelly, D. M. 2003, AJ, 125,1210

Alton, P. B., Davies, J. I., \& Bianchi, S. 1999, A\&A, 343, 51

Bruzual, G., \& Charlot, S. 2003, MNRAS, 344, 1000

Colbert, J. W., et al. 1999, ApJ, 511, 721

de Grijs, R., O'Connell, R. W., \& Gallagher, J. S., III. 2001, AJ, 121, 768

Draine, B. T., \& Bertoldi, F. 1996, ApJ, 468, 269

Engelbracht, C. W., Gordon, K. D., Rieke, G. H., Werner, M. W., Dale, D. A., \& Latter, W. B. 2005, ApJ, 628, L29

Förster Schreiber, N. M., Genzel, R., Lutz, D., Kunze, D., \& Sternberg, A. 2001, ApJ, 552, 544

Förster Schreiber, N. M., Genzel, R., Lutz, D., \& Sternberg, A. 2003a, ApJ, 599, 193

Förster Schreiber, N. M., Sauvage, M., Charmandaris, V., Laurent, O., Gallais, P., Mirabel, I. F., \& Vigroux, L. 2003b, A\&A, 399, 833

Freedman, W. L., et al. 2001, ApJ, 553, 47

Heckman, T. M., Armus, L., \& Miley, G. K. 1990, ApJS, 74, 833

Heckman, T. M., Lehnert, M. D., Strickland, D. K., \& Armus, L. 2000, ApJS, 129,493

Helou, G., Lu, N. Y., Werner, M. W., Malhotra, S., \& Silbermann, N. 2000, ApJ, 532, L21

Helou, G., et al. 2004, ApJS, 154, 253

Hoopes, C. G., et al. 2005, ApJ, 619, L99

Irwin, J. A., \& Madden, S. C. 2006, A\&A, 445, 123

Jarrett, T. H., Chester, T., Cutri, R., Schneider, S. E., \& Huchra, J. P. 2003 , AJ, 125,525

Kennicutt, R. C., Jr., et al. 2003, PASP, 115, 928

Laurent, O., Mirabel, I. F., Charmandaris, V., Gallais, P., Madden, S. C., Sauvage, M., Vigroux, L., \& Cesarsky, C. 2000, A\&A, 359, 887

Lehnert, M. D., Heckman, T. M., \& Weaver, K. A. 1999, ApJ, 523, 575

Leitherer, C., et al. 1999, ApJS, 123, 3

Loiseau, N., Nakai, N., Sofue, Y., Wielebinski, R., Reuter, H.-P., \& Klein, U. 1990, A\&A, 228, 331

Lu, N., et al. 2003, ApJ, 588, 199
Martin, C. L. 1998, ApJ, 506, 222

McCrady, N., Graham, J. R., \& Vacca, W. D. 2005, ApJ, 621, 278

McLeod, K. K., Rieke, G. H., Rieke, M. J., \& Kelly, D. M. 1993, ApJ, 412, 111

Nakai, N., Hayashi, M., Handa, T., Sofue, Y., Hasegawa, T., \& Sasaki, M. 1987, PASJ, 39, 685

Noriega-Crespo, A., Moro-Martin, A., Carey, S., Morris, P. W., Padgett, D. L., Latter, W. B., \& Muzerolle, J. 2004, ApJS, 154, 402

Normand, P., Rouan, D., Lacombe, F., \& Tiphène, D. 1995, A\&A, 297, 311

Pahre, M. A., Ashby, M. L. N., Fazio, G. G., \& Willner, S. P. 2004, ApJS, 154, 235

Reach, W. T., et al. 2005, PASP, 117, 978

Rieke, G. H., Lebofsky, M. J., Thompson, R. I., Low, F. J., \& Tokunaga, A. T. $1980, \mathrm{ApJ}, 238,24$

Rieke, G. H., Loken, K., Rieke, M. J., \& Tamblyn, P. 1993, ApJ, 412, 99

Roche, P. F., Aitken, D. K., Smith, C. H., \& Ward, M. J. 1991, MNRAS, 248, 606

Rodríguez-Rico, C. A., Viallefond, F., Zhao, J.-H., Goss, W. M., \& Anantharamaiah, K. R. 2004, ApJ, 616, 783

Satyapal, S., Watson, D. M., Pipher, J. L., Forrest, W. J., Greenhouse, M. A., Smith, H. A., Fischer, J., \& Woodward, C. E. 1997, ApJ, 483, 148

Shopbell, P. L., \& Bland-Hawthorn, J. 1998, ApJ, 493, 129

Smith, J. D. T., et al. 2004, ApJS, 154, 199

Storey, P. J., \& Hummer, D. G. 1995, MNRAS, 272, 41

Strickland, D. K., Heckman, T. M., Colbert, E. J. M., Hoopes, C. G., \& Weaver, K. A. 2004, ApJS, 151, 193

Sturm, E., Lutz, D., Tran, D., Feuchtgruber, H., Genzel, R., Kunze, D., Moorwood, A. F. M., \& Thornley, M. D. 2000, A\&A, 358, 481

Tacconi-Garman, L. E., Sturm, E., Lehnert, M., Lutz, D., Davies, R. I., \& Moorwood, A. F. M. 2005, A\&A, 432, 91

Walter, F., Weiss, A., \& Scoville, N. 2002, ApJ, 580, L21

Werner, M. W., Uchida, K. I., Sellgren, K., Marengo, M., Gordon, K. D., Morris, P. W., Houck, J. R., \& Stansberry, J. A. 2004, ApJS, 154, 309

Yun, M. S., Ho, P. T. P., \& Lo, K. Y. 1994, Nature, 372, 530 


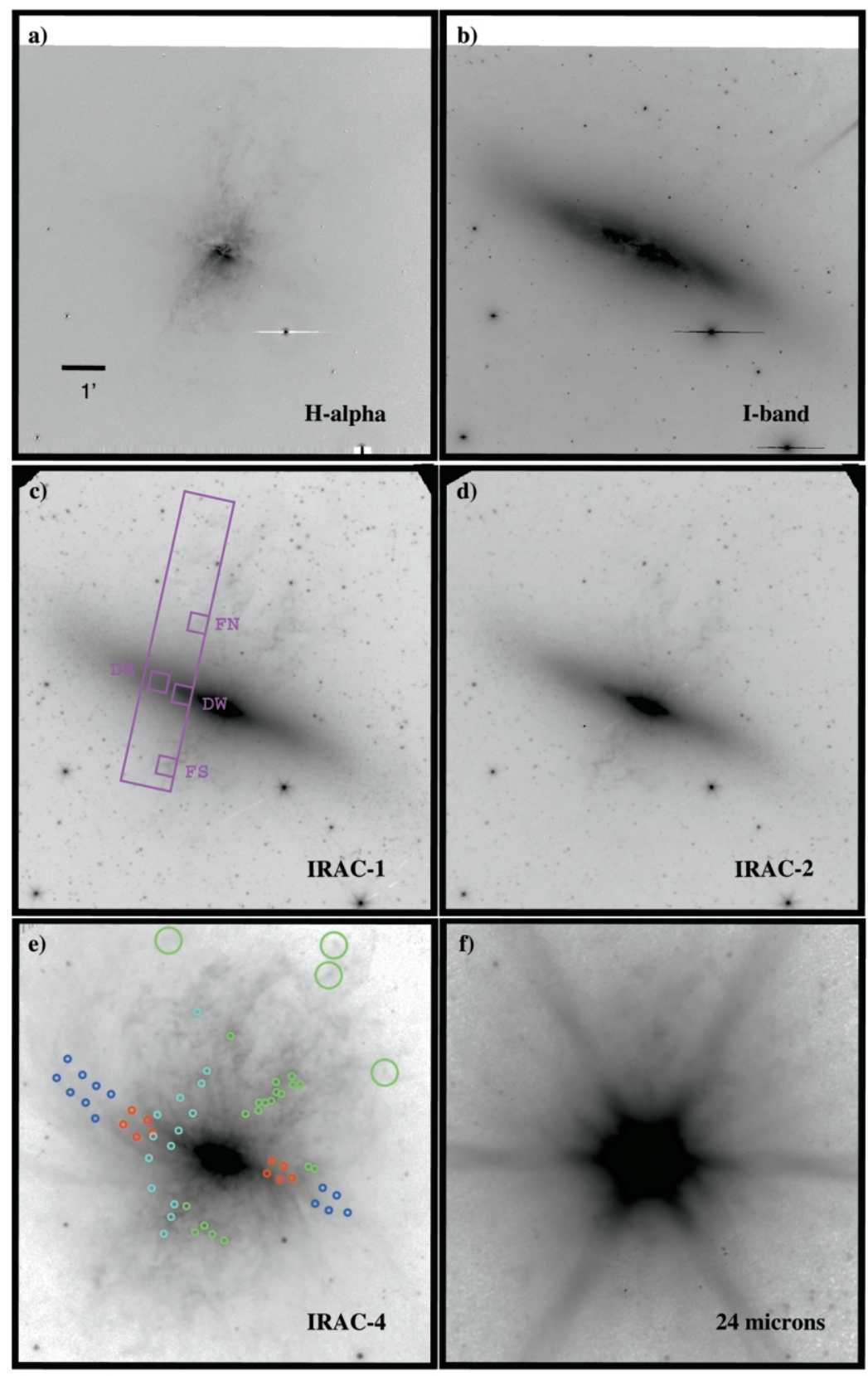

PLATE 1

FIG. 1.-Images of M82, displayed with a logarithmic stretch. The images are (a) $\mathrm{H} \alpha,(b) I$ band, $(c) 3.6 \mu \mathrm{m},(d) 4.5 \mu \mathrm{m},(e) 8.0 \mu \mathrm{m}$, and $(f) 24 \mu \mathrm{m}$. Each panel is $9.2 \times 10.2$ on a side, or $9.6 \times 10.7 \mathrm{kpc}$ at the adopted distance to M82 of $3.6 \mathrm{Mpc}$. Sizes and locations of photometry apertures are indicated in $(e)$, while the extent of the IRS spectral map and the positions of the four spectroscopic apertures are labeled in $(c)$. 


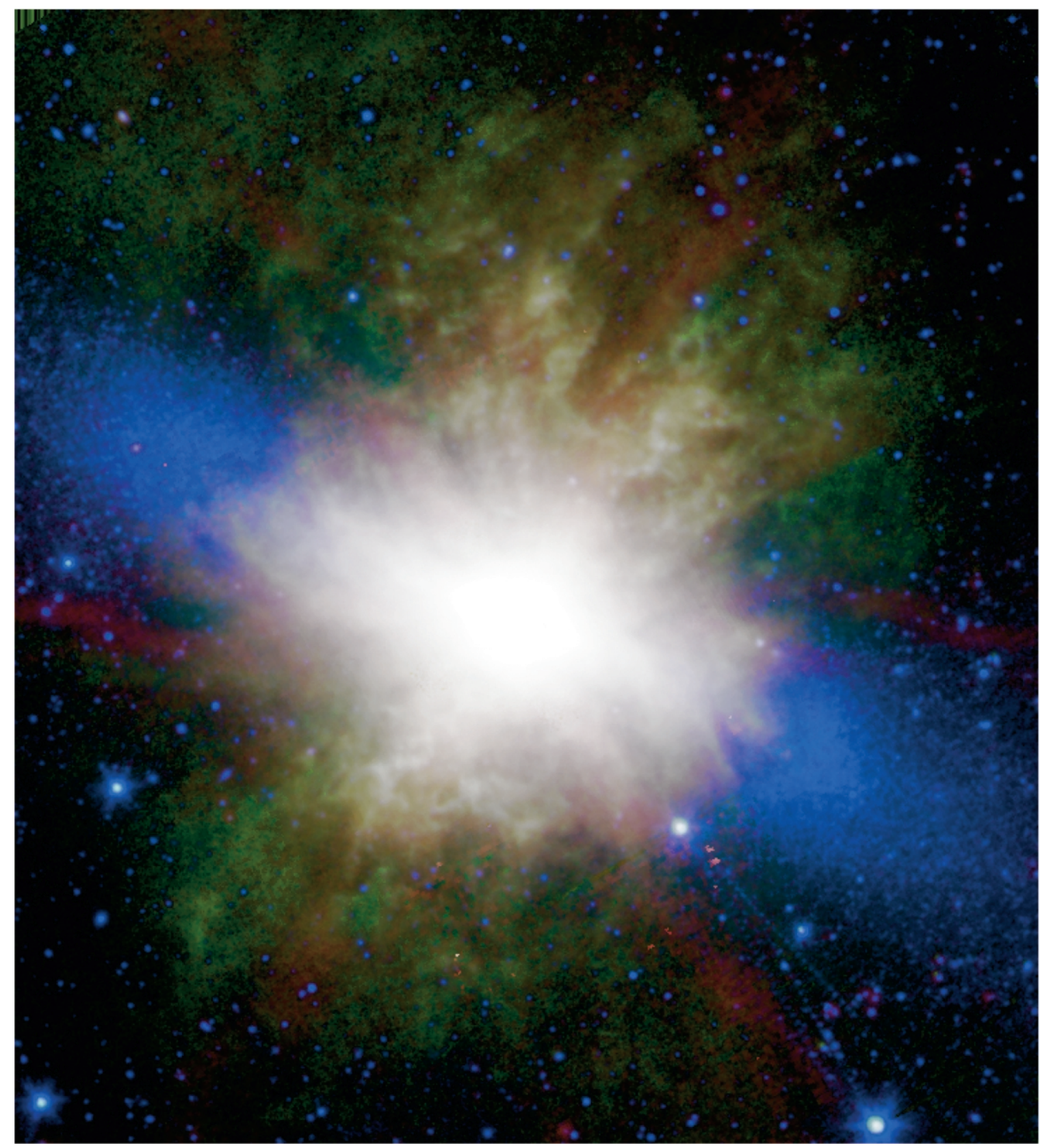

PLATE 2

FIG. 3.-Color-composite image of M82, displayed with a logarithmic stretch. The blue channel is the IRAC band 1 image at $3.6 \mu \mathrm{m}$ and the green channel is the IRAC band 4 image at $8.0 \mu \mathrm{m}$, while the red channel is the MIPS band 1 image at $24 \mu \mathrm{m}$, where the bright nucleus and associated diffraction spikes have been subtracted as described in $\S 2$; the radial red streaks are residuals of the subtraction. The angular extent of the image is the same as the panels in Fig. 1. 